\title{
Atrio-Ventricular Dissociation in Meningococcal Meningitis
}

\author{
Maj J Etherington \\ MRCP (UK), RAMC
}

Senior Registrar Rheumatology and Rehabilitation

Maj J Salmon

MRCP, (UK) RAMC

Senior Registrar Medicine

\section{Brig G Ratcliffe}

MSc, FRCP, Late RAMC

Consultant Physician

Queen Elizabeth Military Hospital, Woolwich, London SE184QH

SUMMARY: A case is presented of meningococcal meningitis, without septicaemia, which was associated with a transient atrio-ventricular dissociation. The need for cardiac monitoring in similar cases is discussed.

\section{Introduction}

The heart can be affected by meningococcal disease in a variety of ways. In septicaemia, there may be direct involvement of the myocardium and its conducting system by the organism or by immune complex deposition. In uncomplicated meningitis, the meningococcus may exert a distant influence, possibly through alterations in sympathetic tone.

Meningitis, with or without septicaemia, may lead to dysrhythmia and bradycardia, necessitating cardiac monitoring in all cases.

\section{Case Report}

An 18 year old Officer Cadet presented to his Medical Officer with a two week history of a mild pyrexial illness. He complained of headache, insomnia, and a productive cough. Treatment was with Asprin, oral Erythromycin and admission to the observation ward of the medical centre.

Later that evening he deteriorated, developing a macular rash, severe headache, photophobia and neck stiffness. He was transferred to hospital where his level of consciousness fell rapidly. On examination there was a tachycardia but blood pressure was normal and the chest was clear. Cranial nerves were intact, fundoscopy normal, reflexes bilaterally brisk and plantars upgoing.

Lumbar puncture produced turbid cerebrospinal fluid (CSF) at an opening pressure of $55 \mathrm{~cm}$ of water. He was started on intravenous Benzyl Penicillin and Chloramphenicol whilst laboratory confirmation of a bacterial meningitis was awaited.

Full blood count revealed a neutrophil leucocytosis with a total white cell count of $16.7 \times 10 \mathrm{~g} / \mathrm{l}$. Two sets of blood cultures, one taken before the administration of antibiotics, were negative. Urea and electrolytes were within normal limits. Examination of the CSF showed a protein content of $7.65 \mathrm{~g} / \mathrm{l}$, with greater than 1000 white cells $/ \mathrm{cm}^{3}$, predominantly polymorphs. There were Gramnegative diplococci, some intracellular, confirmed later asō a Group B Meningococci.

During admission the patient developed a bradycardia of 55 beats per minute but there was no evidence of cardiovascular compromise. The blood pressure remaining stable at $130 / 90$ and the urine output constant-o A twelve lead electrocardiogram (ECG) showed a variable atrio-ventricular (AV) dissociation with an average atrial rate of 50 and ventricular rate of 57 complexes per minute. The cardiac axis was +75 degrees. There were no other ECG changes. An echocardiogram was normal.

Over the subsequent 24 hours the heart rate varied between 55 and 85 beats per minute with persisting AV dissociation. The patient's circulation remained stable and his rhythm needed no treatment.

His condition improved generally and he became apyrexial within 48 hours. The AV dissociation persisted for 72 hours after admission before reverting spontaneously to sinus rhythm. An ambulatory 24 hour ECG was performed one week later which showed normal sinus rhythm throughout. A twelve lead ECG was normal.

The patient, and his close contacts, were treated with Rifampicin as a prophylactic measure and he was fit for discharge, with no neurological or cardiovascular sequelae, twelve days after admission.

\section{Discussion}

This case reminds us of the rapid and potentially fatal deterioration which may occur in meningococcal meningitis. The care shown may be criticised, as there was a delay between diagnostic suspicion and the 
administration of appropriate antibiotics, a procedure which should occur at the primary care level, before transfer. Furthermore, it may be argued that the patient should have received a cephalosporin antibiotic until the confirmation of a purely meningococcal infection. However the case does illustrate a potential cardiac complication of Meningococcal meningitis occurring in the absence of septicaemia. Although the effects of meningococcaemia and other forms of septicaemia on the myocardium have been described, there is only one previously reported case of Atrio-ventricular (AV) dissociation in an unidentified, partially treated, meningitis (1).

In this context the term septicaemia refers to the systemic infection of a patient after local invasion from a mucosal colony leads to bacteraemia. Bacteraemia is also a prerequisite in a localised infection of the meninges but, as in the case presented, there may not be evidence of systemic involvement. Where there is evidence of septicaemia, direct involvement of heart tissue by the organism in the form of myocarditis or endocarditis may occur. The latter may cause abscess formation or necrosis in the interatrial septum with consequent destruction of the AV junction or the bundle of His. A case of fatal complete heart block has been recorded as a result of abscess formation in Streptococcus faecalis infection (2). Haemorrhagic myocarditis has been described in postmortem studies of dogs infected with the meningococcus, with intracellular organisms detected in the myocardium of some cases. There is also record of a human case of intermittent complete heart block associated with meningococcus. Postmortem study of this case revealing extensive myocarditis with microscopic foci of necrosis in the conduction system and subendothelial petechiae (3).

When organisms are not present in heart tissue meningococcal endotoxaemia may activate coagulation and platelet thromboses. If situated in mycardial vessels this may lead to ischaemia, necrosis and impaired cardiac function. Later cardiac complications could be the result of immune complex deposition following the appearance of antibodies to the meningococcal polysaccharide antigen at the sixth day after the onset of infection.

Electrocardiographic abnormalities have also been documented with disease confined to the meninges. In one prospective study comparing ECG changes in patients with tuberculous and pyococcal meningitis compared with pyrexial controls, there were frequent abnormalities (4). All of the 8 patients suffering from pyococcal disease demonstrated some form of irregularity. Five cases showed ST segment depression and four prolonged QTc intervals. Elevated ST segments, flat or inverted T waves, broad notched $\mathrm{T}$ waves and a wide QRST angle were also reported. Two cases which demonstrated prominent $U$ waves had fatal outcomes. In tuberculous meningitis ECG abnormalities were present in seven out of ten cases, with six cases showing ST depression. In the control group only three had ST depression and two QTc prolongati角. There was no association between electrolyte inbalan and arrhythmia.

A further study of 31 patients with meningitis show民d that $74 \%$ had evidence of ECG changes, principaty notched $\mathrm{T}$ waves in the precordial leads (1). There was also evidence of conduction defects, with 3 patien $\overrightarrow{\text { ss }}$ suffering complete heart block and the one case of AVt dissociation. The latter occurred in a partially treatesd bacterial meningitis, where the presence of a septicaen was not known. ECG changes were more common $\overrightarrow{\text { का }}$ those with bacterial meningitis (15 out 16) compared fo viral or aseptic meningitis ( 8 out of 15 ). In the contfol group, with pneumonia, only five out of 60 showed $E \vec{C}$ changes.

Although there appears to be a high incidence of ECSI abnormality, the patho-physiology of cardi complications in disease confined to the meninges unclear. Altered ECGs have been reported in intracranial events such as subarachnoid and intra-cerebrll haemorrhage, head injury and space occupying lesions. The ST segment and T waves are often involved with QTc prolongation and prominent $U$ waves, similar $q_{0}$ meningitis. It was suggested by Shanker that the changes might be mediated by abnormalities in sympathetic foñe secondary to a rise in intracranial pressure, and tha reason why Tuberculous disease had fewer ECG seqpeg was because pressure rose more gradually in 1 iq $s$ situation.

By monitoring five patients with raised intracron of pressure, using a pressure transducer in the extrafuigl space and comparing readings with two patients of nowion pressures, a correlation between raised intracr pressure and ECG abnormality was described by Jach et al (5). However, the features were not entirely simifor to those found in meningitis. All patients with raise pressure had abnormal $\mathrm{T}$ waves, flat, notched or tall. was suggested that tall $\mathrm{T}$ waves were an early feature of raised pressure. Two had depressed ST segmen Prominent $U$ waves were associated with pressures abovie $30 \mathrm{mmHg}$. The QT intervals became shorter as press rose except in the one case of a severe rise, above $1 \overline{\bar{\alpha}} 0$ $\mathrm{mmHg}$, when it became prolonged. Surprisingly, given the above features, Mehta found no association betwegen ECG changes and opening pressure of cerebro-spinal fl童d on lumbar puncture (1).

What then is the significance of these ECG finding The reports would suggest that the changes in E\&G morphology are not associated with maligna dysrrhythmia. However, in a study of $200 \mathrm{fagal}$ meningococcal infections $80 \%$ had evidence of acte myocarditis with occasional evidence of focal necro $\mathrm{g}$ (6). It may be that cardiac involvement is a latent causeñf death in this disease. Aderka (7) reported a high incidence of potentially life threatening arrhythmias duriqg septicaemia in patients with a prior history of myocardial infarction. Previous cardiac disease may, therefore 
predispose to malignant dysrhythmia in the presence of septicaemia.

Although, Dillman et al (8) found cardiac involvement more commonly in meningococcaemia than in uncomplicated meningitis (11 abnormalities out of 14 cases of meningococcaemia compared to two out of six in the meningitis group) it is clear that cardiac involvement is a risk in any meningococcal disease. Whether there is direct involvement of the myocardium or alterations in sympathetic tone, cardiac involvement may be an overlooked cause of sudden death in any form of meningitis, particularly in patients with pre-existing cardiac disease. In view of these findings we would suggest that cardiac monitoring should be performed in all cases of meningitis to detect and treat dysrrhythmias in combination with the early antibiotic treatment of the disease.

\section{REFERENCES:}

1. Mehta S, Kronzon I, laniado $\mathrm{S}$. Electrocardiographic Changes in Meningitis. Is J Med Sci $1974 ; 10$ (7) : 748-52.
2. KlEID J, Kim E, BRAND B. Heart Block complicating acute bacterial endocarditis. Chest 1972; 61 (3) : 3013.

3. Detsky AS, Salit IE. Complete heart block in Meningococcaemia. Ann Emerg Med 1983; 12 (6): 391-393.

4. Shanker A, Raman R, Raghavan S. ECG changes associated with Meningitis, with special reference to serum electrolyte levels. Indian Heart $J$ 1977; 29 (5): 267-71.

5. JaChuk SJ, Ramani PS, Clark F, Kalberg RM. Electrocardiographic abnormalities associated with $\stackrel{\varnothing}{\Omega}$ raised intracranial pressure. $\mathrm{Br} \mathrm{Med} J$ 1975; 1 (5952): ® 242-4.

6. Hardman JM. Meningococcal infections: A review of 200 fatal cases. I Neuropathol Exp Neurol 1967; 26: 119.

7. Aderka D, Sclarovsky S, Lewin RF. Lifethreatening arrhythmias in septicaemia. Is J Med Sci 1984; 20 (6): 535-7.

8. Dillman CE, MCaweeney WJ, Songster CL. Electrocardiographic evaluation of patients with Meningococcal Infections. J SC Med Assoc 1970; 66 官 (2): $37-42$. 